\title{
West African International Summer School for Young Astronomers
}

\author{
Linda E. Strubbe ${ }^{1}$ and Bonaventure Okere ${ }^{2}$ \\ ${ }^{1}$ Science Teaching \& Learning Fellow, University of British Columbia, \\ 6224 Agricultural Road, Vancouver, British Columbia, V6T 1Z1, Canada \\ email: linda@phas.ubc.ca \\ ${ }^{2}$ NASRDA Centre for Basic Space Science, \\ Nsukka, Nigeria \\ email: ibokere2001@yahoo.com
}

The West African International Summer School for Young Astronomers (WAISSYA) is a week-long program for university science students and teachers from West Africa to develop their interest in astronomy. The first summer school was held in Abuja, Nigeria, in 2013; the second Summer School was held in Nsukka, Nigeria, in July 2015. West Africa has a large number of students interested in science, but a paucity of facilities or interest from funding bodies in developing West African astronomy. Our broad goals for the WAISSYA program are: (1) to introduce West African students to astronomy; (2) to exchange ideas about teaching and learning in West Africa and abroad; and (3) to continue building a sustained astronomy partnership between West Africa and Canada. We now briefly describe three defining aspects of WAISSYA 2015.

- We designed our curriculum from evidence-based teaching strategies, emphasizing active learning and scientific thinking (e.g., Wieman 2014, NRC 2012, Chinn \& Malhotra 2002). The curriculum included a two-day hands-on inquiry activity on the Cosmic Distance Ladder, interactive lectures with group discussions and multiple-choice voting, and a project where students designed their own astronomy teaching module to teach at their home institutes after the Summer School.

- The instructional team comprised astronomers from Canada, Nigeria, Germany, and Gabon. We held a workshop for instructors before the school, with goals of sharing evidence-based teaching strategies, and building collaboration and sustainability in the team. Instructors formed international pairs who collaborated to design their lessons using the teaching strategies we modeled and discussed in the instructor workshop. This paradigm builds on research on paired teaching for professional development in other contexts (e.g., Henderson et al. 2009, Stang \& Strubbe 2015).

- We are evaluating outcomes of the Summer School in several ways. Students took the Colorado Learning Attitudes about Science Survey (Adams et al. 2006) and a set of astronomy concept inventory questions (e.g., Hufnagel 2002) at the beginning and end of the Summer School. Students wrote daily reflections about their learning, and also completed a school evaluation survey. We collected reflections from the instructors as well. All of these data are currently being analyzed.

The next WAISSYA will be held in Accra, Ghana, in 2017.

We are pleased to acknowledge the WAISSYA 2015 teaching team: R. Eze, W. Kerzendorf, V. Murray, T.D.C. Nguyen, F. Odoh, P. Okouma, E. Sudum, J. Zhang, and the authors Linda E. Strubbe and Bonaventure Okere. We are grateful for funding and support 
from: the IAU Office of Astronomy for Development, the Dunlap Institute for Astronomy and Astrophysics, the Canadian Institute for Theoretical Astrophysics, the NASRDA Centre for Basic Space Science, the European Southern Observatory, and the University of British Columbia. 